\title{
3D Finite-element Analysis of Soil Seepage in Water-rich Gravel Stratum
}

\author{
Jiangle $\mathrm{Li}^{1,2^{*}}$, Sheliang Wang ${ }^{1}$, $\mathrm{Li} \mathrm{Gao}^{1}$, Yanzhou Hao ${ }^{1,2}$, Shirong Zhang ${ }^{1}$ \\ ${ }^{1}$ Xi'an University of Architecture and Technology, Shaanxi 710055, China \\ ${ }^{2}$ Henan University of Urban Construction, Pingdingshan 467036, China
}

Corresponding Author Email: 30080206@hncj.edu.cn

https://doi.org/10.18280/ijht.370221

Received: 15 November 2018

Accepted: 2 March 2019

\section{Keywords:}

water-rich gravel stratum, $3 D$ seepage field, subway foundation pit, seepage features

\begin{abstract}
Our research targets a subway foundation pit construction project in Nanning, a city in southwestern China. The foundation pit was excavated in the water-rich round gravel stratum. The theoretical calculation was combined with finite-element analysis, which is based on drag coefficient method, to derive the relevant seepage parameters of the stratum. The complex seepage surface was solved through the meshing of virtual grids with fixed nodes. The 3D seepage field around the ground-connecting wall in the pit (hereinafter referred to as the wall) was investigated through finite-element analysis on midas GTS NX. In this way, the author identified the features and law of soil seepage around the wall in the water-rich gravel stratum. The results show that the middle and lower part of the wall were within the sand layer and the gravel layer, both of which are strongly permeable; the seepage rate around the wall peaked in the middle of the wall $(0.156 \mathrm{~m} / \mathrm{sec})$; there was an obvious seepage effect in the pit; the waterrich gravel stratum had greater seepage water inflow than any other stratum; the pore water migration features could be described accurately by the seepage control equation of the heterogeneous anisotropic porous medium.
\end{abstract}

\section{INTRODUCTION}

The water-rich gravel stratum is a widely distributed, thick unfavorable geological formation. The stratum has coarse particles, poor uniformity, loose structure and strong permeability. Depending on gradation, the stratum can be divided into fine gravel layer (particle size: $1 \sim 10 \mathrm{~mm}$ ), medium gravel layer (particle size: $10 \sim 100 \mathrm{~mm}$ ) and coarse gravel layer (particle size: $>100 \mathrm{~mm}$ ).

Due to the poor gradation, the water-rich gravel stratum often has remarkable soil seepage [1]. This particular feature poses a severe threat to the safety and stability of underground structures $[2,3]$. If a foundation pit is excavated below the groundwater level, the groundwater will continue to infiltrate into the pit. To keep the pit safe and stable, it is necessary to take measures against discharge, seepage and leakage [4-6].

Therefore, it is important to analyze the law of groundwater seepage at the ground-connecting wall (here in after referred to as "the wall") under high water pressure before foundation pit construction project, considering the seepage features of each soil layer [7-10].

\section{ENGINEERING BACKGROUND}

Our research targets a subway foundation pit construction project in Nanning, a city in southwestern China. The foundation pit was excavated in a narrow strip-shaped basin, which lies in the center of an erosion zone. The project mainly deals with the upper part of sedimentary stratum, consisting of alluvial gravels and soil layers. According to the Geological Survey on Nanning, Guangxi Zhuang Autonomous Region, this type of stratum is widely distributed and has an extremely high seepage. The underlying bedrocks mainly include tertiary mudstone, silty mudstone, argillaceous siltstone and siltstone.

\section{3D SEEPAGE ANALYSIS}

The seepage effect under the pit wall can be measured by the water level difference between the inside and the outside of the wall. It is this effect that produces the penetration force, which undermines the stability of the wall and exerts a horizontal pressure on the outside of the wall. If the seepage gradient is too large in local areas, the wall may suffer from seepage deformation and the failure of the waterproof layer.

The drag coefficient method is often adopted to compute the seepage field of underground structures $[11,12]$. However, this method only applies to structures with simple geometry and regular shape. For large, complex underground structures, the drag coefficient method requires many assumptions and predictions, and may lead to a huge error. What is worse, a large amount of empirical results is needed to compute the head of the seepage field.

To disclose the exact seepage features of the water-rich gravel stratum, this paper combines the theoretical calculation and the finite-element analysis and derives the following seepage parameters of the stratum.

The water-rich gravel stratum can be viewed as a heterogeneous anisotropic porous medium [13]. In general, the continuous differential governing equation for stable saturated seepage in such a medium can be expressed as:

$$
-\frac{\partial}{\partial \boldsymbol{x}_{i}}\left(\boldsymbol{k}_{i j} \frac{\partial \boldsymbol{h}}{\partial \boldsymbol{x}_{j}}\right)+\boldsymbol{Q}=0
$$


where, $x_{i}$ is the coordinates of the object, $i=1,2,3 ; k_{i j}$ is the permeability tensor; $h=x_{3}+p / \gamma$ is the total water head; $x_{3}$ is the position water head; $p / \gamma$ is the pressure head; $Q$ is term of source and sink.

The various boundary conditions can be defined as:

$$
\begin{gathered}
\boldsymbol{h} / \Gamma_{1}=\boldsymbol{h}_{1} \\
-\boldsymbol{k}_{\boldsymbol{i j}} \frac{\partial \boldsymbol{h}}{\partial \boldsymbol{x}_{j}} \boldsymbol{n}_{\boldsymbol{i}} \mid \Gamma_{2}=\boldsymbol{q}_{\boldsymbol{n}} \\
-\boldsymbol{k}_{\boldsymbol{i} \boldsymbol{j}} \frac{\partial \boldsymbol{h}}{\partial \boldsymbol{x}_{\boldsymbol{j}}} \boldsymbol{n}_{\boldsymbol{i}} \mid \Gamma_{3}=0 \text { and } \boldsymbol{h}=\boldsymbol{x}_{3} \\
-\boldsymbol{k}_{\boldsymbol{i j}} \frac{\partial \boldsymbol{h}}{\partial \boldsymbol{x}_{\boldsymbol{j}}} \boldsymbol{n}_{\boldsymbol{i}} \mid \Gamma_{2} \geq 0 \text { and } \boldsymbol{h}=\boldsymbol{x}_{3}
\end{gathered}
$$

where, $h_{1}$ is the known head function; $n_{i}$ is the cosine of the normal direction at the seepage boundaries, $i=1,2,3 ; \Gamma_{1}, \Gamma_{2}$ and $\Gamma_{3}$ are the first factor of the seepage boundary, the second factor of the seepage boundary and the transition surface of the seepage gradient, respectively; $\Gamma_{4}$ is the overflow surface of the transition surface of the seepage gradient; $q_{n}$ is the normal flow. The out flow direction is positive.

Generally, the calibration of gradient transition surface is not considered during the calculation of the pressure seepage field. Since the set of solutions is the seepage domain, denoted as $\Omega_{1}$ [14], the soil seepage feature can be derived inversely through only one iteration.

Concerning the non-pressure seepage flow with gradient transition surface, it is very difficult to determine the parameters like the shape of the transition surface, the location of the critical surface and the actual area of the seepage area, adding to the difficulty of numerical calculation.

Currently, the popular way to solve the problem is to combine numerical analysis with conventional algorithms. Nevertheless, the seepage field of actual pit foundation construction project may be difficult to solve by conventional algorithms, due to the irregular transition surface of the seepage gradient. After all, the geological conditions and drainage system are often extremely complex. To overcome the defect, this paper puts forward a method to solve complex seepage surface by meshing the transition surface into virtual grids with fixed nodes

The meshing was carried out with a fixed maximum number of nodes and fixed maximum unit aspect ratio. Then, the actual seepage area $\left(\Omega_{l}\right)$ was corrected continuously through iteration based on the virtual seepage area $\left(\Omega_{2}\right)$. In this way, the parameter variation of the seepage field can be mitigated. The iterative control equation of this method can be expressed as:

$$
\boldsymbol{K} \boldsymbol{h}=\boldsymbol{Q}-\boldsymbol{Q}_{2}+\Delta \boldsymbol{Q}
$$

where, $K, h$ and $Q$ are the total conduction matrix of the whole calculation domain $\left(\Omega=\Omega_{1}+\Omega_{2}\right)$, the head coefficient of the boundary node and the equivalent seepage parameter; $Q_{2}$ is the equivalent seepage parameter of each node; $\Delta Q=K_{2} h$ is the seepage parameter of the node of the singular field covering unit and the flowing unit.

\section{FINITE-ELEMENT MODEL}

\subsection{Modeling}

This section sets up a finite-element model of the pit wall in the water-rich gravel stratum on the software midas GTS NX, aiming to disclose the seepage features of the local soil. The $3 \mathrm{D}$ seepage field calculation targets the soil layer, whose thickness is 2.5 times that of the wall on the left side, right side and the underside. In total, the established model contains 67,557 nodes and 125,148 grids. Since the wall dimension is much greater in the longitudinal direction than the lateral direction, this paper only takes account of the soil seepage along the longitudinal direction. The parameters of the soil surrounding the wall were configured according to the geological survey. The finite-element model is shown in Figure 1 below.

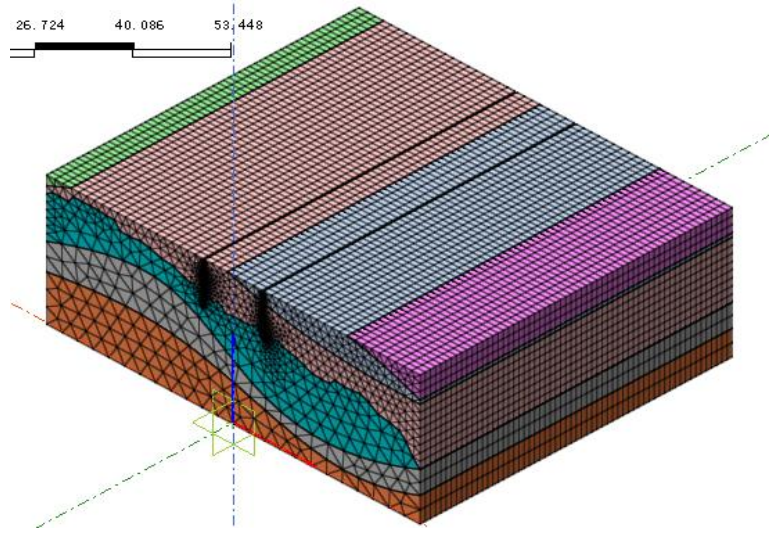

Figure 1. The 3D finite-element model

During finite-element analysis, the grid size is negatively correlated with the computing accuracy. Here, the area with the most significant impact on seepage is simulated with five different levels of grid size, ranging from 0.1 to $1 \mathrm{~m}$. As shown in Figure 2, the results were contrasted by the stress concentration at the foot of the wall.

Three working conditions were considered in our analysis, namely, the construction of discharge channel around the pit, the slotting of the wall, and the construction of the wall and joint. The three working conditions occurred consecutively. The outcome of the previous working condition is the initial state of the following working condition.

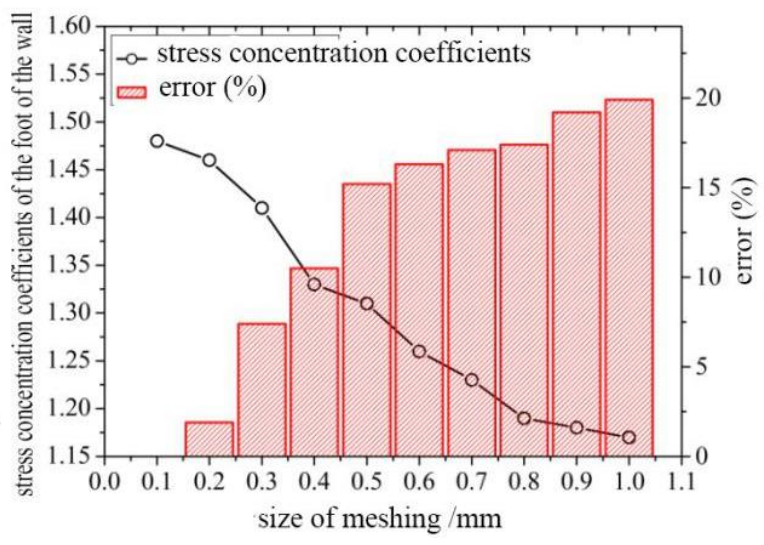

Figure 2. Effects of different grid sizes on computing accuracy 


\subsection{Results of numerical analysis}

Figures 3 present the numerical results on soil seepage around the pit wall of the subway station in the water-rich gravel stratum.

It can be seen from Figure 3 that the mudstone layer and the weathered rock layer at the bottom of the wall formed an antiseepage system for the inverted funnel. The permeability coefficient was maintained in the range of approximately 0.06 $\mathrm{m} / \mathrm{sec}$. The middle and lower part of the wall were within the sand layer and the gravel layer, both of which are strongly permeable. As shown in Figure 3, the finite-element analysis shows that the seepage rate around the wall peaked in the middle of the wall $(0.156 \mathrm{~m} / \mathrm{sec})$. It can be seen from the computing results that the gravel formation had the greatest groundwater seepage rate.

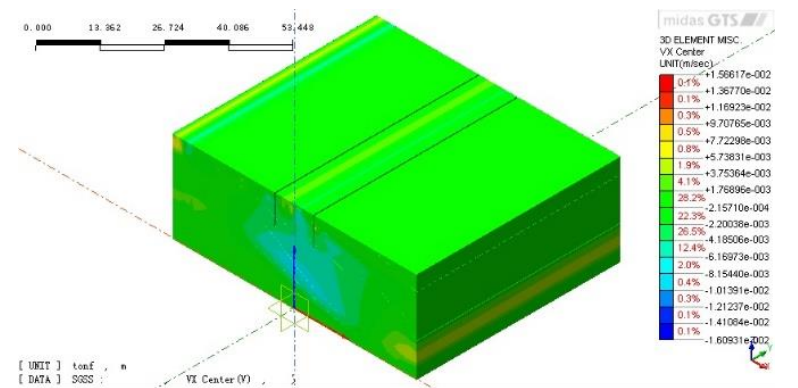

(a) Overall

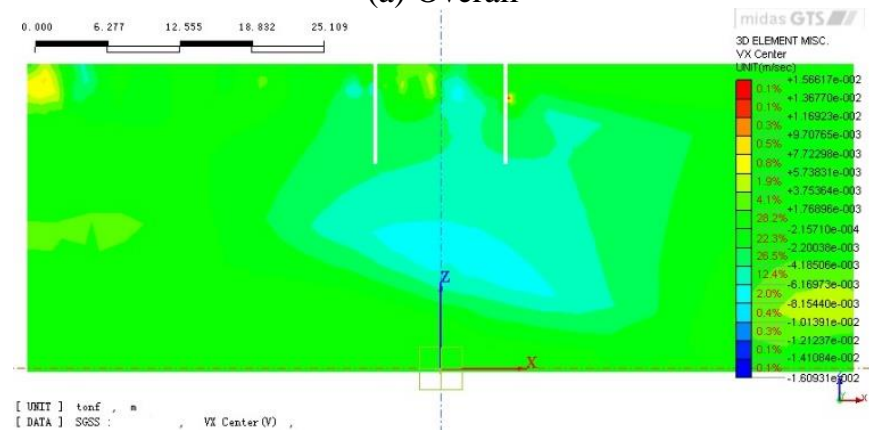

(b) Front

Figure 3. Soil seepage in the x-direction

As for the discharge effect, the seepage rate of each layer in the pit was much lower than that in the soil around the pit. This means the wall can greatly reduce the seepage damage of the soil in the pit and enhance the stability of the soil around the wall. The study area is rich in water, as evidenced by the abundance of groundwater and high water level. The safety of the foundation pit is mainly threatened by groundwater inrush, i.e. the water inflow through seepage. Therefore, the author analyzed the soil seepage flow around the pit wall and plotted the results in Figures 4 below.

The above analysis shows that the water inflow inside and outside the pit peaked respectively at $2.9 \times 10-2 \mathrm{~m}^{3} / \mathrm{sec}$ and $4.2 \times 10-2 \mathrm{~m}^{3} / \mathrm{sec}$. Two obvious water inrush zones appeared on the interface between the sand layer and the gravel layer after the pit wall was completed. The maximum water inflow was not observed around the wall, thanks to the wall's waterproof effect. The significant concave curve on the wall demonstrates that the wall clearly prevented the groundwater inrush from the sand layer and the gravel layer.

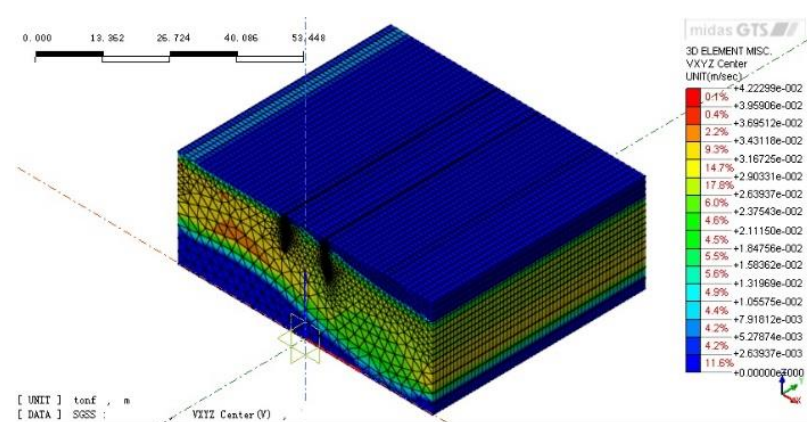

(a) Overall

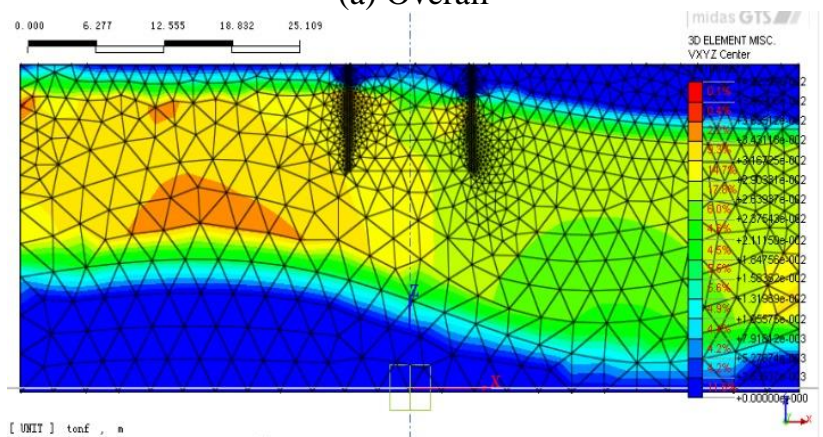

(b) Front

Figure 4. Soil seepage flow around the pit wall

As shown in Figures 5, the longitudinal permeability of surface water gradually declined with the growth in soil thickness, and started to increase gradually when it reached the head of confined groundwater.

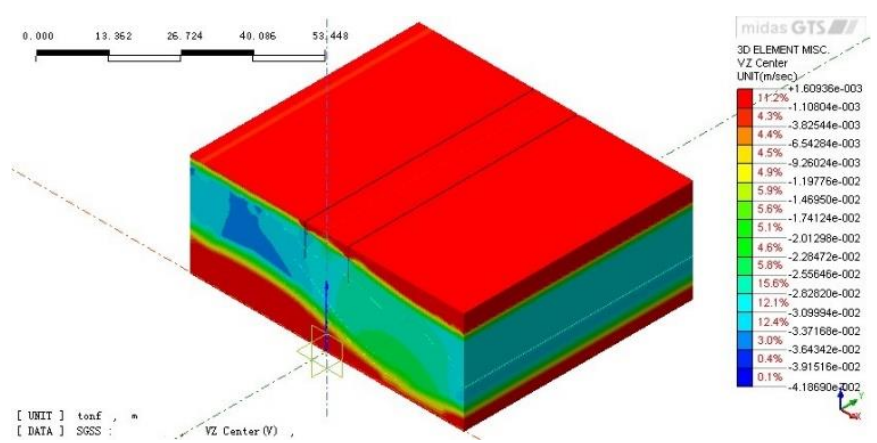

(a) Overall

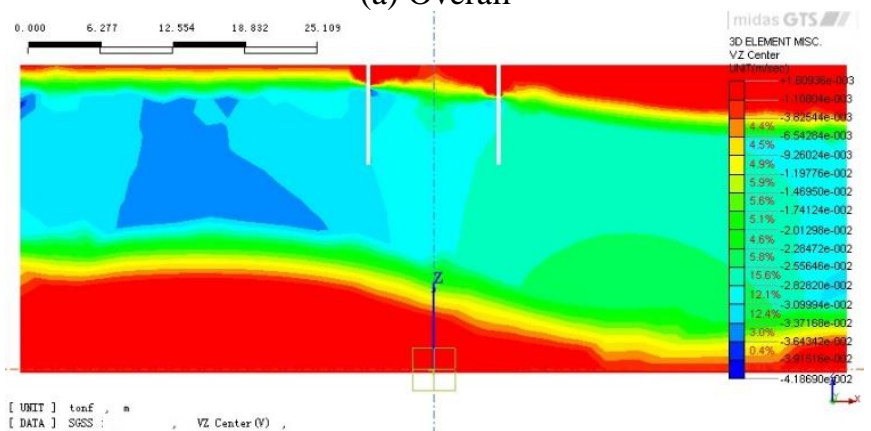

(b) Front

Figure 5. Soil seepage in the longitudinal direction

The above analysis indicates that the longitudinal seepage of the soil in the pit was greater than the seepage of the soil outside the pit. The main reason lies in the fact that the seepage water can only develop downward under the lateral constraint of the pit wall, which does not change with the soil depth. When the seepage water reaches the foot of the wall, the lateral 
constraint disappeared, and the seepage strength of the soil in the pit gradually approaches that of the soil outside the pit.

\section{CONCLUSIONS}

The research object is the deep foundation pit of a subway in China, excavated in the water-rich gravel stratum. The 3D seepage field around the pit wall was studied through theoretical analysis and numerical simulation, aiming to disclose the law and features of soil seepage. The following conclusions were drawn through the analysis.

(1) Considering the grading features of the soil in the water-rich gravel stratum, the pore water migration features could be described accurately by the seepage control equation of the heterogeneous anisotropic porous medium. The complex seepage surface was meshed to virtual grids with fixed nodes, and employed to solve the parameters of the seepage field iteratively, without causing significant variability.

(2) Our analysis shows that the mudstone layer and the weathered rock layer at the bottom of the wall formed an antiseepage system for the inverted funnel. The permeability coefficient was maintained in the range of approximately 0.06 $\mathrm{m} / \mathrm{sec}$. The middle and lower part of the wall were within the sand layer and the gravel layer, both of which are strongly permeable. The seepage rate around the wall peaked in the middle of the wall $(0.156 \mathrm{~m} / \mathrm{sec})$.

(3) The maximum water inflow was not observed around the wall, thanks to the wall' $\mathrm{s}$ waterproof effect. The significant concave curve on the wall demonstrates that the wall clearly prevented the groundwater inrush from the sand layer and the gravel layer.

(4) The 3D finite-element analysis of soil seepage reveals that the water-rich gravel stratum had greater seepage water inflow than any other stratum. Under the surface water seepage and the restraint of the wall, the foundation pit of the subway saw an obvious seepage effect, calling for more efficient discharge method for the deep foundation pit of subway in the water-rich round gravel stratum.

\section{REFERENCES}

[1] Ren, G.H. (2014). Case study on consolidation of waterrich cobble/gravel strata in nanning by means of soletanche grouting. Tunnel Construction, 34(12): 11831190. http://dx.chinadoi.cn/10.3973/j.issn.1672741X.2014.12.011

[2] Bejan, A. (2015). Constructal thermodynamics. Constructal Law \& Second Law Conference, Parma, pp. S1-S8.

[3] Chen, X.Y., Tan, Z.S., Yuan, J., Wang, G., Li, J.W.
(2017). Case study on soil reinforcement method for the cross-passage construction of shield tunnel in water-rich round gravel stratum. China Civil Engineering Journal, 50(S1): 105-110.

[4] Yang, Y., Tan, Z.S., Peng, B., Li, J.P., Wang, G. (2017). Study on optimization boring parameters of earth pressure balance shield in water-soaked round gravel strata. China Civil Engineering Journal, 50(S1): 94-98.

[5] Lei, W. (2015). Research on the environment impact and control countermeasures induced by dewatering confined water of Suzhou metro. Southeast University.

[6] Xie, X.Y., Wang, Q., Liu, H., Li, J., Qi, Y. (2016). Settlement control study of shield tunnelling crossing railway. Chinese Journal of Rock Mechanics and Engineering, 35(S2): 521-531.

[7] Liu, T.Y., Zhang, W., Tan, C., Ma, Z.F. (2018). Numerical simulation of gravity dam seepage field based on UDEC - with Datengxia hydropower station as an example. Environmental and Earth Sciences Research Journal, 5(4): 101-106. https://doi.org/10.18280/eesrj.050404

[8] Wei, Y., Wang, L., Yang, G.S. (2018). Temperature field distribution of a freeze sinking shaft under seepage conditions in cretaceous formation of Western China. International Journal of Heat and Technology, 36(3): 1055-1060. https://doi.org/10.18280/ijht.360336

[9] Yan, H. (2016). The study of information control about construction of deep foundation pit in soft soil. Wuhan: Hubei University of Technology.

[10] Yan, X.Q. (2014). Experimental study on soft soil micropore structure characteristics and permeability properties Guangzhou: South China University of Technology.

[11] Ning, S.C. (2015). Analysis of the seepage characteristics and stability of colluvial soil highway slope in Dabie Mountain. South China University of Technology.

[12] Xu, Y.S., Ma, L., Shen, S.L., Sun, W.J. (2012). Evaluation of land subsidence by considering underground structures that penetrate the aquifers of Shanghai, China. Hydrogeology Journal, 20(8): 16231634.

[13] Pool, M., Carrera, J. (2010). Dynamics of negative hydraulic barriers to prevent seawater intrusion. Hydrogeology Journal, 18(1): 95-105. https://doi.org/10.1007/s10040-009-0516-1

[14] An, N., Chen, H.Z., Yu, X.J., Huang, C.B. (2016). An immersed Crouzeix-Raviart finite element method for anisotropic flow models in porous media. Numerical Mathematics, 38(1): 24-41.

[15] Hou, X., Sun, W. (2018). Calculation of seepage spill point position based on total potential energy in real domain. Chinese Journal of Rock Mechanics and Engineering, 40(4): 612-617. 